\title{
Nudos para los cambios prescriptivos en materia de sexualidad en Chile: la doble moral como instrumento de dominación y la reconfiguración político-institucional de la Iglesia Católica.
}

Knots prescriptive changes in sexuality in Chile: the double standard as a means of domination and political-institutional reconfiguration of the Catholic Church.

\section{ESAU FIGUEROA*}

Resumen: Chile ha sido catalogado como un país moderno en lo económico pero conservador en lo valórico al demostrar dificultades para abordar legalmente temas relacionados con la sexualidad, situación que ha complejizado su trayectoria normativaprescriptiva. Este artículo plantea una aproximación general de este importante problema a través del análisis de algunas situaciones que podrían incidir en su desarrollo. Para ello se presentan dos discusiones empírico-teóricas conceptualizadas como "nudos" para los cambios normativos en materia de sexualidad. En lo concreto, se abordan los problemas de la doble moral como instrumento de dominación y la reconfiguración institucionalnormativa de la Iglesia Católica y su nuevo alcance político-prescriptivo, concebidos ambos como nudos y medios para comprender de mejor forma la complejidad del fenómeno de estudio.

Palabras Clave: Sexualidad, normatividad, nudos, cambios.

Abstract: Chile has been listed as a modern country economically but conservative in what valórico to prove legally difficult to address issues related to sexuality, their situation has become more complex policy-prescriptive path. This article presents a

\footnotetext{
* Esau Figueroa: Docente Universidad del Bío-Bío, Concepción, Chile. Código ORCID: org/00000002-1040-0433 Contacto: efigueroa@ubiobio.cl
} 
general approach to this important issue through the analysis of some situations that could affect their development. For this, two empirical and theoretical discussions conceptualized as "knots" for policy changes in sexuality are presented. In particular, problems of double standards as an instrument of domination and institutional-policy reconfiguration of the Catholic Church and its new political-prescriptive scope, both conceived as knots and means to better understand the complexity of the phenomenon addressed, I study.

Keywords: Sexuality, regulations, knots, changes.

\section{Introducción}

$\mathrm{D}$ esde la década de los 90 Chile ha logrado posicionarse en la región latinoamericana como un país moderno y pujante, proceso iniciado luego de recuperada su democracia y fundado en una economía de libre mercado. No obstante, y de forma paradójica a su condición de país próspero ${ }^{1}$, también se ha destacado como un lugar conservador en relación con temas que involucran debates valóricos ${ }^{2}$ (Informe Mori World Values Survey, 2006).

Los problemas del campo de la sexualidad han sido tradicionalmente ubicados en esta categoría, lo cual alude generalmente a un debate moral entre posturas: una esencialista, naturalista y fundada en la familia tradicional heterosexual y otra, de carácter constructivista, sujeta a un referente subjetivo e histórico de diversidad cultural y social (Valdés y Guajardo, 2007).

La transición desde una sociedad tradicional hacia una moderna, entendida desde una concepción durkhemiana, implica un conjunto de cambios del orden moral, en los que la sexualidad está presente. Las transformaciones de carácter económico, político, sociocultural, que han afectado a Chile (Joignant y Güell, 2009) dan cuenta de este proceso, en el cual es posible identificar tensiones normativas que exponen la existencia de una sociedad en plena mutación.

El desarrollo de la normatividad prescriptiva en materia de sexualidad en Chile se ha constituido como una de las expresiones más claras de estas tensiones. Si se revisa la historia de cada uno de los proyectos legislativos que han buscado generar cambios en esta materia será posible comprobar como cada uno de ellos ha implicado extensos e intrincados procesos de discusión, cuestión que ha llevado al país a una situación de

\footnotetext{
${ }^{1}$ Denis Altman (2006) hace alusión a la relación directa entre sociedades más ricas y mayores niveles de libertad valórica o moral.

${ }^{2}$ Los valores pueden ser entendidos como un tipo de conocimiento social cuya función es facilitar la adaptación de los individuos a su entorno. Son jerárquicos, pues los hay superiores e inferiores (dependiendo de cada tema y situación), además de bipolares, debido a que en tanto exista un valor también habrá un contravalor (Carrasco y Osses, 2005).
} 
inequidad y atraso en relación a otros países de la región, tales como Argentina, Uruguay o Brasil. Un ejemplo al respecto fue la inexistencia en Chile hasta 2004 de una ley de divorcio, lo cual hizo del país uno de los dos últimos lugares en el mundo sin contar con una legislación de este tipo. Del mismo modo, vale la pena recordar que solo recién en el año 1999 se despenalizó la homosexualidad.

Aun así, y si bien se ha mantenido la complejidad asociada a la generación de normatividad prescriptiva, no se pueden desconocer los avances y cambios ocurridos en variados temas relacionados con el género y la sexualidad ${ }^{3}$. No obstante, la situación sigue siendo problemática y precaria en cuanto al resguardo y vulneración tanto de los derechos humanos como de los sexuales y reproductivos ${ }^{4}$ (DDSSRR). En este sentido, son varios aun los problemas que no han encontrado una salida legislativa, como es el caso del aborto $^{5}$, penalizado en todas sus formas en Chile desde 1989. Del mismo modo, y no obstante el potente trabajo llevado a cabo por las organizaciones de la diversidad sexual, el prolongado debate legislativo permitió recién en octubre de 2015 los primeros acuerdos de unión civil (AUC) ${ }^{6}$, mientras que el proyecto de identidad de género se encuentra estancado en el congreso. Otros problemas, en tanto, ni siquiera han tenido oportunidad de legitimarse o discutirse como asuntos públicos, como es el caso del comercio sexual.

Así mismo es importante aclarar que la resolución prescriptiva de estas problemáticas no siempre debe entenderse como una "solución", ya que, de hecho, en ocasiones, la misma normatividad se ha constituido como objeto de conflicto, evidenciando así un problema instalado más allá de la mera discusión legislativa. Esto es lo que ha ocurrido con la política relativa a la entrega de la "píldora del día después" 7 , la cual continúa siendo objeto de polémica ante las críticas de instituciones religiosas como la Iglesia Católica ${ }^{8}$ y la negativa de ciertos actores políticos a colaborar en su óptima implementación. También reproches han generado la ley Antidiscriminación, la que, si

\footnotetext{
${ }^{3}$ Tales como la ya citada despenalización de la homosexualidad con la derogación del artículo 365 en 1999; la promulgación de la ley 18.668 que aseguró la continuidad de estudios para estudiantes embarazadas en 2000; la legalización del divorcio en 2004; la Ley 20.418 que fijó normas sobre Información, orientación y prestaciones en materia de regulación de la fertilidad en 2010; la promulgación de la ley 20.609 antidiscriminación en 2012, entre otros temas.

${ }^{4}$ Entendidos principalmente en el ámbito reproductivo como el "reconocimiento del derecho básico de todas las parejas e individuos a decidir libre y responsablemente el número de hijos... y a disponer de la información y de los medios para ello y el derecho a alcanzar el nivel más elevado de salud sexual y reproductiva. También incluye el derecho a adoptar decisiones relativas a la reproducción sin sufrir discriminación, coacciones ni violencia, de conformidad con lo establecido en los documentos de derechos humanos" (Turner, 2001, p. 209).

${ }^{5}$ Michelle Bachelet incluyó en su programa presidencial en 2013 la posibilidad de legislar un proyecto de despenalización del aborto en tres causales: inviabilidad del feto, riesgo de vida para la madre y violación. ${ }^{6}$ Importante es recordar que el primer proyecto de unión civil fue presentado el año 2003, por lo que el camino para llegar al mencionado AUC ha requerido más de once años.

${ }^{7}$ La píldora del día después alude a un conjunto de medicamentos anticonceptivos tales como acetato de ulipristal, mifepristona y levonorgestrel. Estos se han constituido como un objeto de debate entre sectores que la consideran un mero método anticonceptivo de emergencia y otros que los catalogan como una forma de aborto. Por lo que el centro de la discusión alude al momento en que se produce la concepción.

${ }^{8}$ Históricamente ha sido la Iglesia Católica una institución muy influyente en la política chilena aun cuando la constitución de 1925 consagró la separación entre Estado e iglesia.
} 
bien ha logrado operar dentro de su legalidad, no ha sido capaz de evitar las reiteradas agresiones ni mejorar las condiciones de la población sexualmente diversa9 ${ }^{9}$.

A pesar de lo anterior, la sexualidad de la sociedad chilena ha cambiado. Diversos estudios dan cuenta de tendencias favorables para temas y prácticas que hasta no hace mucho tiempo eran considerados moralmente cuestionables (pero que paradójicamente fueron y continúan siendo problemáticos a nivel prescriptivo), tales como la anticoncepción, las relaciones pre-matrimoniales, el divorcio y el aborto en situaciones de riesgo para la madre o violación, como así también una mayor aceptación de la homosexualidad y una mayor validación de las relaciones sexuales fuera del matrimonio, entre otros temas (Schiappacasse et al., 2004; WorldValuesSurvey, 2006; Dides, Benavente y Sáez, 2010; ICSO, 2013).

El desarrollo de estos cambios podría ser atribuido a una serie de factores como la llegada de la democracia y la consecuente libertad de expresión, la globalización y el acceso a nuevas tecnologías de la información, el crecimiento y los mejores niveles de vida, la progresiva emancipación de las mujeres, la llegada de la modernidad y la consecuente transformación en los sistemas de valores (Bajoit, 2003). Dichos cambios son los que fundamentan la presencia de un cambio cultural ${ }^{10}$ en proceso (Lechner, 2002).

Entonces, si es posible hablar de un cambio cultural, y específicamente, de cambios en las prácticas y percepciones de la sexualidad en Chile: ¿Por qué resulta tan complejo generar cambios legislativos en esta materia?

Se ha dicho que aun cuando Chile ha cambiado en su sexualidad y reproducción (Dides, 2013), la discusión para la resolución de los problemas públicos derivados de estas transformaciones se ha estructurado en función de una disputa ideológica por el resguardo o cambio del orden establecido, involucrando así una cuestión moral.

Respecto de este orden en disputa, la sexualidad y el género han sido tradicionalmente definidos a través de un conjunto de normas y prescripciones vinculadas a un supuesto carácter biológico-esencial, todas ellas construidas para dictar los tipos de comportamiento (femenino-masculino) y las formas de relacionarse que son aceptadas en una sociedad (Lamas, 2013). La fundamentación de estas reglas puede encontrarse en el concepto de sistema sexo-género, el cual designa las disposiciones a través de las cuales una sociedad transforma la sexualidad biológica en productos de la actividad humana (Rubin, 1984).

La modificación prescriptiva de estas disposiciones ha resultado uno de los principales desafíos para grupos feministas y de la diversidad, ya que simbólicamente esta

\footnotetext{
${ }^{9}$ De hecho, el XII Informe DDHH de la diversidad (2013) estima que los asesinatos y agresiones a la población diversa han aumentado en un $33 \%$ y $27 \%$ respectivamente.

${ }^{10}$ De acuerdo con Lechner (2002), el cambio cultural en Chile estaría caracterizado por el paso de la acción colectiva a la estrategia individual propia del consumo, lo cual implica una prevalencia de la identidad individual sobre la colectiva.
} 
normatividad se constituye como un elemento que grafica en gran medida los cambios que progresivamente experimenta una sociedad.

Si una sociedad como la chilena ha cambiado respecto de su sexualidad, y, por tanto, de la forma en que sus integrantes se relacionan entre sí ¿Qué lleva a seguir negando prescriptivamente esta realidad? ¿Qué circunstancias o factores ajenos al cambio cultural en curso inciden en la dificultad para abordar legislativamente estos problemas?

La búsqueda de respuestas ha conllevado a esgrimir desde el sentido común razonamientos simplistas como aquellos que gravitan sobre el "carácter conservador" de la sociedad chilena o su "falta de madurez". Desde posturas académicas se ha argumentado respecto de las relaciones de género ${ }^{11}$ y poder-control (Foucault, 2011) dictadas desde un sistema heteronormado ${ }^{12}$ y androcentrico (Bourdieu, 1998).

La presente reflexión se estructura tanto en términos académicos como políticos, poseyendo dos objetivos básicos.

El primero es visibilizar la dificultad asociada a la generación de cambios prescriptivos (legislación) sobre las normas establecidas en el sistema sexo-género formal vigente en Chile, junto con denunciar las importantes consecuencias sociales que esto ha conllevado.

El segundo es revisar dos discusiones provistas de elementos empíricos y teóricos que permitan comprender mejor las condiciones sobre las que se desarrolla este fenómeno (y sus posibles causas), las cuales han sido conceptualizadas como "nudos" para los cambios normativos en materia de sexualidad en Chile. Tales discusiones corresponden al problema de la doble moral como instrumento de dominación y la reconfiguración institucional-normativa de la Iglesia Católica y su nuevo alcance político-prescriptivo.

Cabe destacar que esta revisión no busca repetir argumentos ya conocidos, sino más bien analizar y enriquecer estas discusiones con el afán de encontrar nuevas claves que permitan abordar de forma crítica este importante problema, el que, si bien ha cobrado mayor notoriedad en el último tiempo, aun se constituye como una deuda pendiente en Chile.

\footnotetext{
${ }^{11}$ El cual cuestiona el orden imperante al entenderlo como una construcción simbólica, establecida sobre los datos biológicos de la diferencia sexual (Lamas, 2013).

${ }^{12}$ Entendida la heteronormatividad como la imposición de un sistema social, político, económico y simbólico fundamentado en el patriarcado y las prácticas sexuales heterosexuales.
} 


\section{La doble moral como instrumento de dominación y continuidad en normativa sexual}

Las normas pueden entenderse como prescripciones formales e informales que permiten a los actores reconocer su entorno social y las reglas colectivas allí establecidas, posibilitando así la convivencia cotidiana en función de lo que cada grupo humano define como normal o ideal (Girola, 2009). El cumplimiento de ellas está determinado por un mandato provisto de fuerza moral (Durkheim, 2001) ante el cual la individualidad debe someterse, tratándose así de "obligaciones observadas y protegidas por el grupo" (Canales, 2012, p. 87).

En el ámbito de la generación de normas se ha dicho que la sexualidad es uno de los campos más importante de reglamentar, ya que su regulación se presenta como posibilidad de constitución de comunidad de seres humanos, y por tanto, de instauración de una convivencia normada (Araujo, 2003). Sin embargo, si se considera que la forma en que los seres humanos vivimos y aplicamos las normas sociales no es consciente, y considerando el régimen androcéntrico-patriarcal vigente, la regulación de la sexualidad (y del género) implicaría de antemano una situación de dominación, producto de lo cual diversos grupos han debido acatar la instauración de un orden simbólico incuestionable y por tanto inamovible (Amoros, 1991).

Lo anterior da cuenta de la dificultad de llegar a pensar y concretar cambios normativos estructurales, ejercicio que requiere un análisis consciente de la norma y el cuestionamiento del contenido moral implícito de esta, más aún cuando ella se sostiene en un conjunto de disposiciones prescriptivas codificadas, las cuales se materializan en la aplicación de la ley.

No obstante, Rita Segato (2004) argumenta que la ley sólo implica la legitimación de una posición dentro de una lucha simbólica, mientras que el curso de los fenómenos no requiere de esta para desarrollarse, tal como ocurre con las uniones gay, el aborto o la prostitución. De hecho, las prácticas sociales en ocasiones traspasan las fronteras impuestas por las normas legales, generándose verdaderos sistemas de acción paralelos. Estos operan en un contexto donde la misma restricción pública de la conducta legitima la posibilidad de su transgresión privada.

Tal es el planteamiento efectuado por Bonnie Shepard (2000) quien identifica una amplia brecha entre los discursos públicos y privados referidos a los DDSSRR, haciendo de su práctica una contradicción operativa en términos sociales, políticos y culturales. En este sentido, se entiende que la normatividad emanada de los discursos públicos (la ley) no da cuenta de la realidad y sus necesidades, frente a lo cual las personas hacen uso de verdaderas "válvulas de escape" que les permiten sobrellevar la norma y ejercer la práctica sancionada en la clandestinidad. 
De esta forma se origina al llamado doble discurso, el que puede ser concebido como "el arte de acomodarse a las normas socioculturales tradicionales y/o represivas en el marco de la vida pública, mientras que en el ámbito privado es frecuente que se haga caso omiso a dichas normas o incluso se transgredan" (Shepard, 2000, p. 5).

El aborto es un ejemplo gráfico de cómo en Chile la norma ha operado alejada de la realidad social. Esto ya que al ser criminalizado desde 1989 en todas sus formas, lo que se ha hecho es minimizar una práctica supuestamente marginal y rechazada a partir de una codificación legal.

Esta presunción, sin embargo, es objetada frente a planteamientos de expertos como Ramiro Molina ${ }^{13}$, quien sostiene que en Chile se practicaron en 2013 unos 33.000 abortos solo en el sistema público de salud, ignorándose la cantidad total de procedimientos clandestinos (Yáñez, 2014). Esto implica la marginación de un problema social real, una importante situación de inequidad ${ }^{14}$, y lo que puede ser aún más complejo, la alteración de la autopercepción de las personas como sujetos morales.

Este último punto ha sido poco abordado, ya que en general se suele poner atención en las consecuencias mediáticas del doble discurso (como es la práctica clandestina del aborto o la demanda del comercio sexual), más no se escudriña en el efecto sobre los mismos sujetos y las implicancias en el largo plazo que esto tiene.

Y es que la moral no solo se reduce a un conjunto de valores y reglas que permiten a las personas relacionarse con la realidad en la que se desenvuelven (y ser evaluadas por los demás en función del acatamiento de dichas normas), sino que también influye en la relación de estas consigo mismas en su calidad de "sujetos morales", cuestión que determina la capacidad de subjetivación y por ende, su desarrollo como individuos (Foucault, 2012).

Lo anterior postula que una norma punitiva como la del aborto, al operar funcionalmente sobre un conjunto de estrategias "ilegales" que utilizan las personas para sobrellevarla, obliga a estas últimos a situarse entre territorios moralmente opuestos. Esto supone una contradicción que pone en entredicho la experiencia moral individual y el papel que esta juega en el medio social, lo cual tendría importantes consecuencias en la trayectoria personal y en el desarrollo social.

Respecto de esta situación en el contexto chileno, Kathya Araujo (2003) plantea:

...Los gobiernos chilenos de la última década (1990) tienen entre sus características destacadas haber preservado estas dimensiones contradictorias en las que obligadamente se desarrolla la experiencia moral y ética de las personas en torno a la sexualidad e impulsado a la vez formas de proceder en los sujetos que, dada la contraposición entre la experiencia social y la ley, se ubican en la

\footnotetext{
13 Académico Universidad de Chile y experto en medicina reproductiva.

${ }^{14}$ Ya que los abortos ilegales generalmente son realizados en condiciones de alto riesgo, dependiendo de la capacidad económica para acceder a un mejor (pero siempre ilegal) servicio.
} 
“paradojal situación de estar en un más allá de la ley pero en el corazón mismo de la comunidad" (56).

De este modo, la lógica del doble discurso no solo conlleva a la legitimación de una doble moral, sino también a la instauración de una experiencia moral en constante tensión frente a la perpetuación de políticas que operan paradojalmente bajo el supuesto de una transgresión privada, socialmente aceptada pero públicamente condenada. Esto repercute en el auto-reconocimiento moral de los individuos, distorsionando no solo su dimensión privada, sino también su rol social como ciudadanos.

El problema mayor es que la doble moral disminuye las posibilidades de cuestionar críticamente el contenido y estatus de la norma, por muy represiva o injusta que esta pueda ser, y por tanto de impulsar los procesos de cambio necesarios. Esto ya que un comportamiento basado en dicha lógica posiciona a los individuos en dos situaciones morales: de contradicción, como consecuencia de tener que quebrantar la norma moral que define las relaciones de convivencia con los demás; y de inferioridad, entendiendo que el acatamiento pleno de la norma otorga una superioridad moral que puede ser más poderosa que la ventaja física, económica o tecnológica (Segato, 2004), lo cual expone a esta lógica no como una válvula de escape, sino directamente como un instrumento de dominación, el cual garantiza la perpetuación del sistema sexo-género en curso.

Desde esta perspectiva se podría hipotetizar que la doble moral y su efecto de tensión moral en las personas, ya sea estas protagonistas activas o "cómplices" pasivas ${ }^{15}$, se ha constituido como un importante mecanismo de dominación al favorecer la inmutabilidad del statu quo normativo sexual en Chile. Esto frente a una interacción social y política que se ha habituado al despliegue de estrategias basadas en el doble discurso, cuestión que a su vez disminuye la posibilidad de cambios normativos al impedir el desarrollo del impulso ético (Segato, 2004), concebido este como una herramienta fundamental para abordar críticamente las leyes y la moral sin tener que sufrir por las limitaciones impuestas por el contexto cultural en el que nos hemos formado.

Esto es relevante, pues plantea que los alcances de la doble moral no se limitan al espacio privado donde esta actúa, sino que al tensionar la subjetividad misma de los individuos afecta directamente la capacidad colectiva-pública para cuestionar el marco prescriptivo vigente, paso previo necesario para generar los cambios requeridos. Más aun, dicho problema trasciende lo relativo a la mera normativa sexual, pudiendo determinar la forma en que una sociedad se relaciona políticamente con sus gobiernos, leyes, marcos jurídicos, etc.

Así, este problema no sería exclusivo de Chile, siendo aplicable a cualquier lugar o sociedad que se rija por la experiencia política y social del doble discurso, cuestión que

${ }^{15}$ La tensión moral no solo toca a quien comete la transgresión, sino también a quienes están al tanto de ella, ya sea encubriendo un aborto clandestino, ignorar una infidelidad, saber que un familiar es gay y negarlo, etc. 
requiere el análisis de componentes culturales, idiosincráticos y políticos de cada caso en particular. Al respecto, Latinoamérica se presenta como un lugar interesante considerando su herencia colonial común.

\section{Nudo 2: Las nuevas condiciones para el despliegue de la influencia religiosa en la normativa sexual. El caso de la Iglesia Católica.}

Uno de los argumentos que con mayor frecuencia se cita al momento de hipotetizar sobre las dificultades en Chile para generar cambios legislativos en materia de sexualidad apunta al papel que ha jugado la Iglesia Católica (I.C. $)^{16}$. Sin embargo, algunos estudios locales dan cuenta de una sostenida pérdida de confianza de esta institución en el ámbito social $^{17}$, por lo que resulta interesante comprender en qué medida esta premisa está en lo cierto y cuáles son las condiciones que la han favorecido.

Para comenzar, es necesario confirmar que la influencia de esta institución se ha plasmado en muchas de las leyes y políticas del país, aun cuando la separación constitucional entre Estado e Iglesia rige desde 1925. También es de conocimiento público el carácter conservador de sus valores en relación con temas concernientes a la sexualidad y el género, resumidos estos en una moral destinada a legitimar solo las relaciones conducentes a la procreación (Htun, 2010). Al respecto, Bourdieu (1998) sostiene que esta institución "inculca explícitamente una moral pro-familiar, enteramente dominada por los valores patriarcales, especialmente por el dogma de la inferioridad natural de las mujeres" (p. 107).

Sin ir más lejos, en 2012 el ex Papa Benedicto XVI "denunció" a los estudios de género como un engaño que ha distorsionado la visión misma del ser humano, calificando de "falaces" sus argumentaciones teóricas y exponiéndolos como un peligro para el valor de la familia y la libertad. También el actual Papa Francisco llamó a la "teoría de género" una colonización ideológica y las comparó con la imposición de doctrinas tales como el nazismo y fascismo (Murillo, 2015).

Frente a ello no debiera resultar inesperado constatar que gran parte de las discusiones referidas a cambios legislativos en materia de sexualidad en Chile han encontrado una fuerte resistencia en esta institución. Así, su peso se ha dejado sentir sobre cualquier iniciativa o proyecto que "amenace" con desestabilizar el orden moral tradicional que ella protege, independiente de las necesidades sociales al respecto. Tal

\footnotetext{
${ }^{16}$ Se trabaja en función de la Iglesia Católica, no porque con ello se omita la influencia y rol que han tenido otras instituciones religiosas como las iglesias evangélicas, sino porque su rol en la sociedad y el Estado chileno ha sido tradicional y políticamente más extenso y marcado.

${ }^{17}$ Encuestas como la ICSO han mostrado como su niveles de confianza social en Chile ha bajado desde un 47,5\% en 2009 hasta un 26,7\% en 2014, en medio de lo que se ha establecido como un declive del discurso religioso (Barrientos, 2006).
} 
presión fue la que debieron sortear proyectos como el de despenalización de la homosexualidad, el reconocimiento de los hijos fuera del matrimonio, el divorcio, la educación sexual y más recientemente la ley antidiscriminación ${ }^{18}$. Y son estas mismas objeciones las que deben afrontar actualmente discusiones referidas al aborto y al matrimonio igualitario, rechazadas por el vínculo establecido entre sexualidadreproducción-heterosexualidad y la consecuente negación de cualquier desarrollo sociosexual.

La pregunta que emerge es ¿Por qué en un Estado constitucionalmente laico, una institución de estas características posee atribuciones para objetar cambios prescriptivos a costa de vetar o desplazar problemas públicos legítimos?

Al respecto, Mala Htun (2010) argumenta que, al recuperarse la democracia en países como Chile o Brasil, la I.C. se hizo de un sólido capital político cultivado durante la lucha contra las dictaduras durante los años 70 y 80, al defender los derechos humanos y criticar los regímenes militares. Esto le valió el respeto y consideración por parte de las nuevas elites democráticas, quienes no se atrevieron a contradecir posteriormente sus juicios morales, validando así su influencia en temas de Estado.

Sin embargo, para el retorno de la democracia la I.C. no era la misma de los 70 . Esto debido a que desde la ascensión a papa de Juan Pablo II (1978) se llevaron a cabo diversas reformas conservadoras al interior de la institución, entre ellas la erradicación en Latinoamérica de la teología de la liberación ${ }^{19}$. Con este objetivo se reubicaron figuras religiosas, implantando en las altas jerarquías eclesiásticas autoridades provistas de una moral más cercana a sectores de derecha, quienes velaron por preservar una normatividad más tradicional (Htun, 2010).

Si bien este planteamiento es interesante y coherente respecto de la relación entre I.C. y política, su aplicación es limitada en países de Latinoamérica donde no hubo dictaduras y la influencia de esta institución es poderosa, como es el caso de México. Sin embargo, si es revelador de la trayectoria histórica moderna de dicha institución en la región.

En otra línea, Irma Palma (2008) apunta a una reconfiguración en las relaciones entre sujetos e instituciones normativas, específicamente al desarrollo de estas últimas. La autora establece que una homogeneidad institucional y normativa ha dado paso a una mayor heterogeneidad y la instauración de sentidos contradictorios en diversos temas. Esto plantea que instituciones médicas, religiosas, científicas, culturales en muchos casos "compiten" por imponer su criterio en temas comunes ${ }^{20}$.

\footnotetext{
${ }^{18}$ El Arzobispo de Santiago, Monseñor Ezzati, cuestionó la puesta en marcha de esta ley, pidiendo además al Gobierno en 2013 que no apoyara la Convención Interamericana contra la Discriminación porque esta incluía a la orientación sexual y la identidad de género.

${ }^{19}$ Ideología cristiana que asumía un compromiso social con los pueblos de Latinoamérica, planteando una reflexión a la luz de la fe sobre la praxis histórica (Di Tella, 2001)

${ }^{20}$ Como han sido en temas como la orientación sexual e identidad de género.
} 
Para entender mejor esto es conveniente remitirnos a los planteamientos de Michel Bozon (2004), quien argumenta que la normatividad sexual ha dejado de construirse por disciplinas y controles externos para ser determinada por disciplinas de control interno basadas en las relaciones sociales y la experiencia individual. Al mismo tiempo, la medicalización de la sociedad ha contribuido a que los problemas sexuales dejen de ser considerados morales, transformándose en parte del bienestar individual, social y de salud sexual. No obstante, aun cuando los individuos pueden regular sus necesidades sexuales de forma autónoma, siguen siendo criticados y juzgados socialmente dependiendo de su edad o del hecho si son hombres o mujeres. En otras palabras, la normativa sexual no se libera de controles, sino que los reestructura en un contexto marcado por procesos de individualización y subjetivación comunitaria, propios de la modernidad, y en el que la sanción prescriptiva sigue poseyendo gran importancia.

Tal cambio habría llevado a la I.C. a articularse directamente con el propio Estado y el campo de la política para hacer prevalecer su moral frente a la intromisión de otras institucionalidades, como la de la salud, por ejemplo. En otras palabras, a partir de dicho momento, la I.C. ya no opera doctrinalmente sobre "sus fieles" o la sociedad como había hecho durante siglos, sino que comienza a actuar sobre ellos a través de la fuerza de la ley, ya que "el campo en que ello se realiza es el de la relación con el Estado, es decir, el poder para transformar la norma en ley” (Palma, 2008, p. 20).

No obstante, el vínculo entre iglesia y política implica en el largo y mediano plazo el establecimiento de nuevas tensiones, las cuales surgen producto del desajuste generado entre los intereses de la I.C. y la ineludible respuesta que debe procurar el sistema político frente a las transformaciones sociales, las cuales muchas veces contravienen la posición de esta institución, quien a su vez presiona sobre las decisiones legislativas. Ejemplos al respecto han sido los procesos previos a políticas como la de prevención del VIH-SIDA ${ }^{21}$, constantemente objetada, y leyes como la de divorcio o antidiscriminación, largamente prolongadas en su discusión legislativa.

Aun cuando este planteamiento es bastante útil para comprender los problemas que deben enfrentar los cambios normativos en Chile, existe la posibilidad de analizar el rol de la I.C. desde una perspectiva complementaria, como es la postulada por Eric Fassin (2010), quien dice que la menor presencia del Estado en Latinoamérica permite que instituciones como la I.C. adquieran mayor importancia y por tanto puedan influir en

\footnotetext{
21 Si bien la Ley 19.779 (2001), salvaguarda los derechos de las personas, asegurando un diagnóstico, control y tratamiento adecuados, como así también resguardando cualquier forma de discriminación, tanto en términos de acceso al tratamiento como también para las personas que viven con el virus, las implicaciones morales-sexuales del VIH continúan presentando desafíos para una política pública óptima a nivel educativo y comunicacional. La controversia se ha centrado desde 1992 en las campañas televisivas y en las que dos canales, Megavisión y Universidad Católica de Chile Televisión, han presentado constantes problemas para su difusión, incluso negándose a transmitirlas ya que estas promoverían las relaciones prematrimoniales, la promiscuidad y la homosexualidad, entre otros valores contrarios al conservadurismo moral.
} 
temas de sexualidad y la vida en general de las personas, al contrario de lo que ocurre en Europa:

Sabemos que en Francia la contraconcepción es algo normal, el aborto es relativamente normal (lo que no quiere decir que la gente piense que está bien, pero muchas personas recurren a él). La Iglesia Católica no influye sobre las prácticas sexuales en Francia y en Europa... la iglesia dice lo que quiere sobre la sexualidad, la gente escucha con oído distraído, y hace otra cosa" (Fassin, 2010, p. s.n).

Esto apuntaría directamente a la conformación política del contexto latinoamericano y las transformaciones gatilladas por la instauración de una lógica de mercado, la cual en el caso particular de Chile ha sido pionera en favorecer un modelo de individuación desprovisto de un componente moral autónomo (Lechner, 2002), cuestión vinculada directamente a carencias como la ausencia de educación sexual, la cual incluso ha sido dejada al arbitrio del mercado ${ }^{22}$.

Esto, junto a la interacción de una variable más amplia como es la importancia cultural que posee la familia en Latinoamérica, permitiría el predominio institucional de la I.C. como garante y reguladora moral del comportamiento político, y a través suyo, social, legitimando su intromisión en debates vinculados a leyes y políticas públicas, especialmente aquellas que abordan temas relativos al género y la sexualidad ${ }^{23}$. En este sentido Fassin señala: "Entre más Estado haya, habrá más individuo, y no porque el Estado conduzca la conciencia de la gente o la adoctrine, sino porque las condiciones para existir en tanto individuo están ligadas a esta fuerza relativa del Estado" (2010, p. s.n).

De este modo, es posible ordenar algunas ideas o hipótesis que podrían dar cuenta del actual rol de la I.C. en la normatividad sexual en Chile:

Primero: establecer que la I.C. ya no ejerce su influjo doctrinariamente sobre la sociedad, viendo mermada su influencia frente a la proliferación de una heterogeneidad de institucionalidades normativas, incluida la propia experiencia subjetiva de los individuos, que compiten por imponer su criterio en lo referente a moral sexual.

Segundo: ante esta situación, la I.C., sacando partido de su trayectoria histórica y política, ha buscado actuar directamente sobre el Estado para hacer prevalecer su doctrina en contenido y la aplicación de la ley.

22 Desde 2010, y por política de Estado, la educación sexual fue dejada en manos de privados, quienes administran los distintos programas a los que pueden acceder los establecimientos educacionales, si es que estos cuentan con los recursos necesarios.

23 Fassin también especifica que tal injerencia religiosa, no obstante, resulta especialmente contradictoria en Estados que se consideran democráticos, ya que la I.C. (y para el caso chileno, también la evangélica) asume la existencia de verdades por encima de la historia que poseen características inmutables (Dios), cuestión que va en contra de los principios democráticos que implican la construcción conjunta de la organización social. 
Tercero, frente a las condiciones políticas y sociales generadas por la implantación del sistema neoliberal del mercado, lo cual se traduce en el debilitamiento del propio Estado y la constitución moral autónoma de los sujetos, la I.C. ha accedido a un remozado espacio de poder político-prescriptivo, compitiendo desde allí con relativo éxito frente a las demás institucionalidades normativas e interfiriendo en la generación de cambios en materia de sexualidad.

En otras palabras, la I.C. continúa influyendo en la normatividad sexual "indirectamente" sobre la sociedad y "directamente" sobre la ley, aprovechando el repliegue del Estado frente al sistema neoliberal y, paradójicamente, gracias a las propias transformaciones morales de la sociedad.

Necesario es plantear también como un análisis comparativo de esta lógica podría dar cuenta en alguna medida respecto de las diferencias entre países cercanos, como es el caso de Chile y Argentina, cuyas trayectorias en normatividad sexual hoy presentan diferencias notables, no obstante, comparten un pasado colonial común y presentar una relación entre sociedad e I.C. parecida. Es decir, dependiendo del contexto de estudio, serán factores como la profundización del sistema neoliberal de mercado, las condiciones políticas y los cambios sociales mismos, entre otros, los que podrían determinar la influencia de la moral religiosa sobre la normatividad sexual.

\section{Conclusiones}

$\mathrm{E}^{\prime}$ análisis de los nudos presentados y las hipótesis de trabajo propuestas permiten dimensionar no solo el alcance particular de estas discusiones sino también la intrincada relación entre las variables allí presentes y otros problemas anexos, provistos estos de características históricas, políticas, económicas, culturales, psíco-sociales, etc. Tal situación me lleva a proponer la idea de un conjunto mayor de nudos, conectados entre sí, los cuales formarían una verdadera red tejida en torno a la moral sexual en Chile.

Esto es relevante pues plantea la existencia de un problema más amplio y complejo, el cual se aleja de visiones aisladas o excluyentes respecto de las casusas y condiciones que han hecho de Chile un lugar de difícil trayectoria para los temas morales sexuales, posicionando así el fenómeno desde una perspectiva más bien sistémica.

Otro punto importante alude al comportamiento de los mecanismos de dominación y control, propios del problema en cuestión, los cuales se complejizan a la par de las sociedades modernas, proliferando, innovando y anexando en función del llamado dispositivo de la sexualidad (Foucault, 2012). Esto implica un constante movimiento de variables involucradas, lo cual me lleva a atribuir un carácter dinámico a la estructura que conforma la referida red de nudos. 
Lo anterior supone la existencia de nudos más estables o de mayor data, tales como la herencia histórico-cultural de la sociedad chilena o las limitaciones normativas de la composición ideológica de la matriz política del país, etc. Del mismo modo es posible identificar otros nudos más recientes, como las implicancias de la administración biopolítica de la población o la forma en que la herencia dictatorial ha condicionado la permeabilidad social frente a los movimientos sociales, etc.

Volviendo al análisis de los nudos, ha sido su acción lo que ha permitido la inmutabilidad de un status quo heredado desde tiempo de la conquista de América, periodo en que el choque producido entre la rígida moral religiosa europea y una sexualidad indígena desprovista de dichas restricciones tuvo como consecuencia la adopción de un comportamiento social cínico y segregador. Este consideró el disfrute de los placeres carnales y la presencia de ciertas formas de vida (indígenas, sodomitas, prostitutas, mujeres, etc.) como cuestiones asociadas a menores niveles de civilización y normalidad, argumento que dio origen a una temprana puesta en escena de la doble moral.

El contenido básico de este planteamiento continúa vigente, siendo custodiado, defendido e impuesto por sectores y actores sociales y políticos influyentes, entre los que destaca la I.C. Esto se evidencia, por ejemplo, a través del precoz vínculo entre la influencia normativa de esta institución y el despliegue de la doble moral, de cuya interacción se ha nutrido un sistema sexo-género basado en la autoridad patriarcal, una concepción funcional-reproductiva de la sexualidad y la marginación total de cualquier forma de vida que contravenga estos preceptos.

Tal como se ha buscado demostrar en el análisis, estos nudos han interactuado constantemente con otros problemas (potenciales nudos), agregando mayor complejidad al fenómeno.

Comprender este carácter relacional junto a la diversificación de los mecanismos de dominación resulta fundamental para entender no solo las dificultades que se han debido sortear para generar cambios normativos sexuales (la cual radica en la constitución de los propios nudos), sino también para reflexionar respecto del contenido e implicancias que poseen dichas transformaciones, sobre todo cuando pueden ser finalmente implementadas, cuestión que por cierto trasciende al caso chileno.

En este sentido es importante destacar como en ciertas ocasiones los mismos cambios legislativos pueden llegar a constituirse como nuevas expresiones de continuidad para el sistema, operando así el dispositivo de la sexualidad bajo lógicas adaptadas a las nuevas circunstancias. Tal es el caso, por ejemplo, de premisas que señalan los riesgos de la integración social basada en el derecho a excluir incluyendo (Galende, 2010), a propósito de las tensiones surgidas en función de los recientes procesos de inclusión de la diversidad sexual en algunos países de América Latina.

Lo anterior plantea nuevas preguntas: ¿quién define como debe vivirse la sexualidad, y por tanto, que es una apropiada moral sexual? ¿En qué medida es posible depositar en el Estado y el derecho expectativas y responsabilidad al respecto? ¿Qué pasa 
con aquellos derechos y formas de vida que no calzan con los nuevos ordenamientos? Y ¿cómo evitar que los nuevos marcos regulatorios se transformen en nuevos nudos?

Interesante para ahondar en esta reflexión es el argumento presentado por Teresa López (2004), quien discute acerca del Estado moderno y su ineludible responsabilidad para intervenir a favor de aquellos grupos que poseen "necesidades especiales", como han sido calificadas las relativas al género y la sexualidad. Frente a ello, el mundo político ha demostrado "solidaridad y benevolencia" al instaurar diversas nuevas ciudadanías, las cuales permiten a estos grupos ejercer algunos derechos básicos. No obstante, y si bien en el corto plazo es positivo, por ejemplo, permitir que personas del mismo sexo puedan legalizar su unión o que las mujeres no sean criminalizadas por abortar bajo ciertas situaciones, también es cierto que la implementación de dicha lógica podría constituirse como el nudo más reciente de la red.

Esto ya que la creación de ciudadanías especiales no contribuiría a la instauración de una ciudadanía plena, en la que todas las personas cuenten con las mismas libertades, justicia, derechos y obligaciones, independiente de su género, orientación e identidad sexual, etnia, situación socioeconómica, etc. Es decir, la transformación real del sistema sexo-genero requiere de una reflexión general acerca de las condiciones de vida y problemas existentes, la relación entre los nudos que constriñen las posibilidades de cambio y el proyecto integral de sociedad que se busca construir.

Muy por el contrario, Eric Fassin (2012) argumenta que una mala utilización de los cambios normativos en materia de sexualidad (democracia sexual) podría conllevar a diversos nuevos problemas, tales como la profundización de la desigualdad social, por ejemplo.

En ello radica la necesidad de considerar este problema en toda su magnitud, desde sus bases causales hasta las consecuencias de sus potenciales soluciones. Para ello es primordial reconocer sus elementos estructurales, aquí propuestos bajo la forma de nudos y red, y cuya operatoria se encuentra determinada por mecanismos de adaptabilidad que permiten salvaguardar la integridad del sistema sexo-género imperante.

Importante también es profundizar no solo en los nudos particulares que componen esta red, sino también estudiar sus conexiones, de modo de poder trascender el alcance aislado de cada uno de ellos y evitar caer así en reduccionismos que contribuyan a fortalecer los propios mecanismos de dominación.

Como última consideración, es importante dar cuenta de la necesidad de generar mayor producción científica en este campo de investigación, requisito fundamental en la tarea de identificar y analizar nuevos nudos para la comprensión cabal del problema. Solo así se podrán generar cambios normativos sustentables en materia de sexualidad, tanto en Chile como en el resto de los países de la región que asuman este desafío, necesario para la construcción de sociedades provistas de mínimos niveles de equidad, diversidad, justicia y libertad. 


\section{Bibliografía}

Altman, D. (2006). Sexo Global. Barcelona: Editorial Océano.

Amorós, C. (1991). Hacia una crítica de la razón patriarcal. Barcelona: Anthropos Editorial.

Araujo, K. (2003). Discurso público sobre la moral sexual. Santiago: Revista de Crítica Cultural, (27), 54-57.

Araujo, K. (2003). La sexualidad como pasión contemporánea. En: Araujo, Kathya y Ibarra (eds.) (2003). Santiago: Sexualidades y sociedades contemporáneas, 101119.

Bajoit, G. (2003). Todo Cambia. Análisis sociológico del cambio social y cultural en las sociedades contemporáneas. Santiago: Editorial LOM.

Barrientos, J. (2006). ¿Nueva normatividad del comportamiento sexual juvenil en Chile? Santiago: Revista Última Década, 14(24), 81-97. http://www.scielo.cl/scielo.php?pid=S071822362006000100005\&script=sci_artt ext

Bourdieu, P. (1998). La dominación masculina. París: Editons du Seuil.

Bozon, M. (2004). La nouvelle normativité des conduites sexuelles ou la difficulté de mettre en cohérence les expériences intimes. En : Marques, J. (Editor) (2004). Normes et conduites sexuelles. Approches sociologiques et ouvertures pluridisciplinaires. Lovaina: Academia Bruylant.

Canales, M. (2012). La palabra grupal. Sociología de Emilio Durkheim. En: Canales, M., Avendaño, O. y Atria, R. (2012). Sociología. Introducción a los Clásicos. Santiago: Ediciones LOM.

Carrasco, E. y Osses, S. (2005). Estudio del perfil valórico. El caso de los estudiantes de primer nivel de carrera de obstetricia y puericultura de la facultad de medicina de la Universidad de la Frontera. Temuco: Revista Estudios Pedagógicos, 31(1), 732.

Dides, C. (15 de febrero de 2013). Chile ha Cambiado: Sexualidades y Políticas Públicas sin Cuerpo. Santiago: $\quad$ El Mostrador. http://www.elmostrador.cl/opinion/2013/02/15/chile-ha-cambiado-sexualidadesy-politicas-publicas-sin-cuerpo/ 
Dides, C., Benavente, C. y Sáez, I. (2010). Principales Resultados: Estudio de opinión pública sobre Aborto, Brasil, Chile, México, Nicaragua. Programa Inclusión Social y Género. Quito: FLACSO.

Durkheim, E. (2001). Las Reglas del Método Sociológico. Ciudad de México: Fondo de Cultura Económica.

Universidad Diego Portales (2011). Encuesta Nacional Universidad Diego Portales. http://www.encuesta.udp.cl/tag/encuesta-nacional-udp-2011/

Universidad Diego Portales (2013). Encuesta Nacional Universidad Diego Portales. http://www.encuesta.udp.cl/descargas/enc2013-2/tablas-sept-2013.pdf

Fassin, E. (7 de enero de 2010). Matrimonio homosexual: entre la doble moral liberal y el conservadurismo. Ciudad de México: La Jornada. http://www.jornada.unam.mx/2010/01/07/ls-portada.html

Fassin, E. (2012). La Democracia Sexual y el Choque de Civilizaciones. Buenos Aires: Revista Mora, 18.

Foucault, M. (2011). Historia de la Sexualidad I. La voluntad del placer. Madrid: Siglo XXI Editores.

Foucault, M. (2012). Historia de la Sexualidad II. El uso de los placeres. Madrid: Siglo XXI Editores.

Galende, F. (2010). Biopolítica y exclusión. Tentativas sueltas. En: Cassigoli, I. y Sobarzo, M. (Eds.) (2010). Biopolíticas del Sur. Santiago: Editorial ARCIS.

Girola, L. (2009). La cultura del "como si”. Normas, anomia y transgresión en la sociedad mexicana. En: Araujo, K. (Ed.) (2009). ¿Se acata pero no se cumple? Estudios sobre la norma en América Latina. Santiago: LOM Ediciones.

Htun, M. (2010). Sexo y Estado. Aborto, divorcio y familia bajo dictaduras y democracias en América Latina. Santiago: Ediciones Universidad Diego Portales.

Informe de Prensa MORI (2006). World Values Survey 2006 Chile. http://www.prolades.com/cra/regions/sam/chi/mori_2006_chile.pdf

Joignant, A. y Güell, P. (2009). El arte de clasificar a los chilenos. Enfoques sobre los modelos de estratificación en Chile. Santiago: Ediciones Universidad Diego Portales.

Lamas, M. (Compiladora) (2013). El género. La construcción cultural de la diferencia sexual. Ciudad de México: Universidad Autónoma de México. 
Lechner, N. (2002). Los Desafíos del Cambio Cultural. Santiago: Centro de Estudios para el Desarrollo.

Lopez, T. (2004). La mitad del mundo. Ética y crítica feminista. Salamanca: Universidad de Salamanca.

Murillo, C. (20 de enero de 2015). Otra dosis de misoginia papal. La izquierda diario. http://www.laizquierdadiario.com/Otra-dosis-de-misoginia-papal

Palma, I. (2007). Sociedad chilena en cambio. Sexualidades en transformación. Tesis doctoral, Universidad de Chile.

Palma, I. (2008). Las instituciones religiosas en la transformación normativa contemporánea en la sexualidad en Chile. Santiago: Revista Psicología, 18(2), 937.

Rubin, G. (1984). Thinking Sex: Notes for a Radical Theory of the Politics of Sexuality. Carole, V. (Ed.) (1984). Londres: Pleasure and Danger.

Schiappacasse, V., Vidal, P., Casas, L., Dides, C. y Diaz, S. (2004). La salud y los derechos sexuales y reproductivos en Chile: 1999-2003. En: Valdés, T. \& Guajardo, G. (Eds.) (2004). Hacia una agenda sobre sexualidad y derechos humanos en Chile. Quito: FLACSO.

Segato, R. (2004). Antropología y derechos humanos: Alteridad y ética en el movimiento de los derechos universales. Buenos Aires: Serie Antropológica.

Shepard, B. (2000). The "double discourse" on sexual and reproductive rights in Latin America: the Chasm between public policy and private actions. Cambridge: Health Human Rights, 4, 110-43.

Turner, S. (2001). Los derechos sexuales y reproductivos y su incidencia en la esterilización y procreación asistida. Santiago: Revista de Derecho, 12, 207-216.

Valdés, T. y Guajardo, G. (2007). Investigación sobre sexualidad y derechos sexuales en Chile (1990-2002). Santiago: CLAM.

Yañez, C. (9 de noviembre de 2014). ¿Cuántos abortos se realizan realmente en Chile?. Diario La Tercera. http://www.latercera.com/noticia/tendencias/2014/11/659603813-9-cuantos-abortos-se-realizan-realmente-en-chile.shtml 